\title{
UNITY IN DIVERSITY: DISCOVERING TOPICS FROM WORDS Information Theoretic Co-clustering for Visual Categorization
}

\author{
Ashish Gupta and Richard Bowden \\ Centre for Vision, Speech and Signal Processing, University of Surrey, Guildford, United Kingdom \\ $\{$ a.gupta, r.bowden $\} @$ surrey.ac.uk
}

Keywords: Co-clustering, Bag-of-Words, Visual Topic Model

\begin{abstract}
This paper presents a novel approach to learning a codebook for visual categorization, that resolves the key issue of intra-category appearance variation found in complex real world datasets. The codebook of visual-topics (semantically equivalent descriptors) is made by grouping visual-words (syntactically equivalent descriptors) that are scattered in feature space. We analyze the joint distribution of images and visual-words using information theoretic co-clustering to discover visual-topics. Our approach is compared with the standard 'Bagof-Words' approach. The statistically significant performance improvement in all the datasets utilized (Pascal VOC 2006; VOC 2007; VOC 2010; Scene-15) establishes the efficacy of our approach.
\end{abstract}

\section{INTRODUCTION}

Visual categorization is a topic of intense research activity in the computer vision and pattern recognition communities. Despite the progress made in the past decade, a satisfactory model for a visual category is missing. The difficulty lies in the fact that a visual category is not a single entity is a composition of distinct parts. These parts could be considered as categories themselves. This contributes to significant variation in appearance of a category. The high intracategory appearance variation implies low-level features associated with a semantically relevant part of the category are scattered over feature space. A hardpartitioning algorithm like Learning Vector Quantization (LVQ) is unable to cluster these scattered features together. This is the key issue that limits the ability of the standard 'Bag-of-Words' (BoW) approach (Csurka et al., 2004), which uses LVQ, to build a codebook of 'visual-words' that can effectively model complex visual category data. Consequently, this paper focuses on a novel approach that can cluster these scattered features together, thereby providing a succinct and robust codebook of 'visual-topics' that can better model complex category data.

The basic concept behind our approach can be visualized in figure 1. BoW partitions feature space into disjoint regions and builds a codebook of 'visualwords' using centroids of these regions. Two descriptors from the same part of a category but located far apart in feature space will be assigned to different

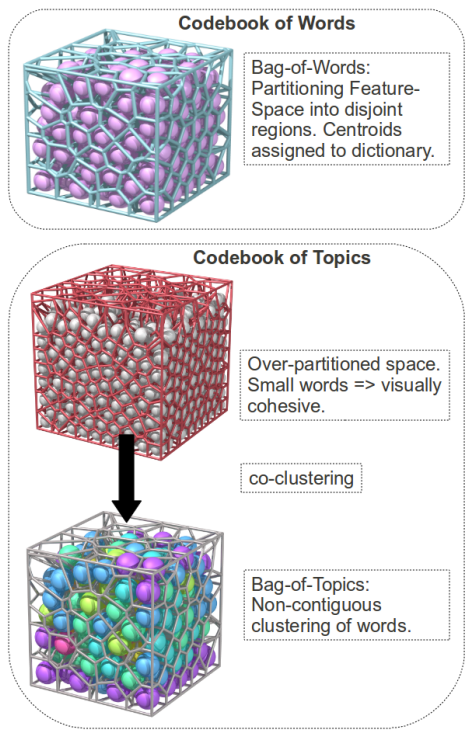

Figure 1: Topic discovery from Words: semantically equivalent but visually distinct words are grouped together. The words of the same color in the bottom cube depict words that have been grouped together, the color represents a topic. Images are public domain samples generated by $3 \mathrm{D}$ tessellation software Voro++, for details see (Rycroft et al., 2006)

'words'. The method we propose groups the 'words' into which these descriptors fall and assigns them to a 'visual-topic', thereby creating a codebook of 'topics'. This is depicted by regions in different parts of space being of the same color, where color denotes topic. This approach can also be considered as non- 
contiguous clustering. The key insight is that clustering of descriptors should be jointly based on two criteria: distribution density in feature space; distribution across images. We utilize co-clustering which optimally clusters on the joint distribution of these criteria to discover topics from words.Some of the earliest work on co-clustering can be found in (Hartigan, 1972). It was further developed in the field of bioinformatics for gene sequence clustering described in (Cheng and Church, 2000). It was introduced as a tool for data mining in (Dhillon et al., 2003) and in (Dhillon, 2001). It was utilized in a computer vision application in (Liu and Shah, 2007).

Our main contributions to this work are:

- We propose a novel approach to discovering visual topics from visual words using information theoretic co-clustering.

- We thoroughly evaluate our approach against the standard BoW approach and show consistent and statistically significant improvement in classification performance.

- We analyze the relevance of codebook size to discover an optimal size for the datasets considered.

- We explored the relation between our approach and different types of visual categories to discover special applicability to a specific type of category.

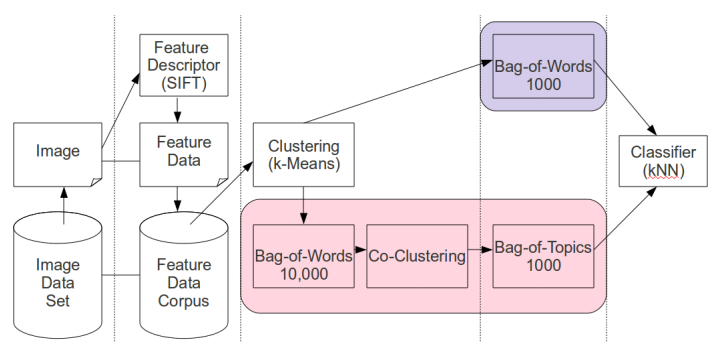

Figure 2: Visual Categorization System: data acquisition, feature extraction, clustering, learning a word codebook, co-clustering, learning a topic codebook, and classification. Blue box has modules of the BoW approach, red box has modules of our approach using co-clustering.

\section{APPROACH}

We first state the motivation for our novel approach and then describe the system and the algorithm. We have formulated our approach based on these insights:

- BoW is based entirely on feature space descriptor density distribution to build a codebook and ignores the distribution density of a codebook element across images.
- Unlike a visual word (particular instance of an category part) a visual topic will almost always occur in a positive sample of the category.

- Feature vectors sourced from a category part are not completely scattered but exist in cliques in feature space.

- Visual words from the same category have similar occurrence distribution statistics across images.

Combining these insights, an algorithm that simultaneously considers: distribution in feature space; distribution across images, when clustering feature vectors should provide a codebook of topics we intend to build. We arrange these two distributions as a image-word data matrix, where the rows are images and columns are words. Co-clustering optimally and simultaneously clusters the rows and column of the data matrix. Therefore we employ it to discover topics. The modules of a typical visual categorization system is shown in figure 2 . The modules in the blue box are used in the traditional approach while the red box contains modules implementing our approach. The remaining modules are common to both approaches. This allows us an effective comparison for both approaches. The steps in our algorithm are:

1. Over-partition feature space: build a huge set of words using an unsupervised clustering technique based purely on descriptor density distribution in feature space.

2. Compute the occurrence histogram of codebook elements. In other words, compute their orderless distribution in the image, for all images in the dataset.

3. Analyze the joint distribution of images and words. We utilize information theoretic coclustering to optimally cluster both images and words. This translates to creation of blocks in the image-word data matrix. The blocks tell us which words are clustered together.

4. Combine the clustered words into topics and create the topic codebook.

\section{LEARNING CODEBOOK}

In this section we discuss how a codebook is learned by the BoW approach and by our approach.

\subsection{Codebook-of-Words}

The mathematical formulation of the BoW model: It encodes visual data in high-dimensional space $\Xi \subseteq$ $\mathbb{R}^{d}$ by a set of code-words $Q=\left\{\psi_{1}, \psi_{2}, \ldots, \psi_{N}\right\}$. 
These code-words are also called visual-words or simply words, $\phi_{i}[i]_{1}^{N} \in \Xi$. The visual data is a set of feature descriptor vectors $\left\{v_{1}, v_{2}, \ldots, v_{M}\right\}$, where $v_{j}[j]_{1}^{M} \in \Xi$. Each visual-word $\psi_{i}$ has an associated section of the high dimensional space $\xi_{i}$. So, the visual data space $\Xi$ is represented by $\left\{\psi_{i}, \xi_{i}\right\}[i]_{1}^{N}$, $\psi \in \mathbb{R}^{d}$, and $\cup_{i}^{N} \xi_{i}=\Xi$. There are several algorithms for computing $\left\{\psi_{i}, \xi_{i}\right\}[i]_{1}^{N}$, which is the codebook. We use a LVQ algorithm which is called as k-means clustering method. The algorithm poses the task as an optimization problem, where the codebook $Q$ is optimized given data $\Upsilon=\left\{v_{1}, v_{2}, \ldots, v_{M}\right\}$ and a distortion measure $D_{\phi}(\cdot, \cdot)$. The distortion measure in an information-theoretic setting using Kullback-Leibler divergence is given as:

$$
D_{K L}(\psi, v)=\left\langle p(v), \log \frac{p(v)}{p(\psi)}\right\rangle
$$

The optimal solution for Kullback-Leibler metric is given as:

$$
\{Q, \Xi\}^{*}=\underset{Q}{\arg \min } L_{K L}\left(Q, D_{K L} \mid \Upsilon\right)
$$

Consider a data-set $\Lambda$ containing $n$ images $\lambda_{i}[i]_{1}^{n}$. An image $\lambda_{i}$ is described by a set of $m$ feature vectors $v_{j}[j]_{1}^{m}$ (number of features $m$ will vary in images). The image $\lambda_{i}$ is encoded by an occurrence histogram of the codebook elements. Each feature vector $v_{j}$ in $\lambda_{i}$ is associated with a visual word $\psi_{k}$. The histogram $h_{\lambda_{i}}$ of image $\lambda_{i}$ is an array $\left\{h_{\lambda_{i}, \psi_{k}}\right\}[k]_{1}^{N}$, where $h_{\lambda_{i}, \psi_{k}}$ is given by:

$$
\begin{aligned}
h_{\lambda_{i}, \psi_{k}} & =\sum_{j=1}^{m} \mathbb{1}_{\psi_{k}, v_{j}} \\
\mathbb{1}_{\psi_{k}, v_{j}} & = \begin{cases}1 & \text { if } v_{j} \in \xi_{k} \\
0 & \text { otherwise }\end{cases}
\end{aligned}
$$

\subsection{Codebook-of-Topics}

Co-clustering attempts to discover topics by analyzing the joint distribution of images and visual words. Visual topic is a selection of visual words that have high occurrence frequency across images. The set of visual words is given by $\{Q, \Xi\}=\left\{\psi_{i}, \xi_{i}\right\}[i]_{1}^{N}$ and the set of visual topics is given by $\{\mathcal{T}, \Xi\}=\left\{\tau_{j}, \zeta_{j}\right\}[j]_{1}^{M}$. Each topic is a cluster of visual words acquired by coclustering. So, a visual topic $\tau_{j}=\left\{\psi_{1}, \psi_{2}, \ldots, \psi_{t_{j}}\right.$ and its associated section of feature space is $\zeta_{j}=\cup_{t=1}^{t_{j}} \xi_{t}$. In the co-clustering scheme all visual words are assigned to some visual topic, and so the union of topic space is the entire feature space, $\cup_{j=1}^{M} \zeta_{j}=\Xi$. To visualize this see figure 1 .

\section{CO-CLUSTERING}

A mathematical formulation of co-clustering: Let $W$ and $Y$ be discrete random variables that take values in sets $w_{u},[u]_{1}^{m}$ and $y_{v},[v]_{1}^{n}$. Here $W$ represents visual word and $Y$ represents image. In the ideal case, the joint distribution $p(W, Y)$ is known. This is the normalized joint distribution of visual words and images. The aim is to cluster $W$ into $k$ disjoint clusters $w_{g},[g]_{1}^{k}$, and $Y$ into $l$ disjoint clusters $\hat{y_{h}},[h]_{1}^{l}$. Let $\hat{W}$ and $\hat{Y}$ denote the corresponding clustered random variables. The information-theoretic approach to optimal co-clustering is to solve:

$$
\min _{\hat{W}, \hat{Y}} I(W, Y)-I(\hat{W}, \hat{Y})
$$

where $I(W, Y)$ is the mutual information between $W$ and $Y$. As shown in (Dhillon et al., 2003), information loss is:

$$
I(W, Y)-I(\hat{W}, \hat{Y})=D(p(W, Y) \| q(W, Y))
$$

where $D(\cdot \| \cdot)$ denotes KL-divergence, and $q(W, Y)$ is given by:

$$
q(W, Y)=p(\hat{W}, \hat{Y}) p(W \mid \hat{W}) p(Y \mid \hat{Y})
$$

It combines both row and column clustering at each step. Row clustering is done by computing proximity of each row distribution, in relative entropy, to certain 'row cluster prototypes'. These are the estimated cluster centroids. Column clustering is carried out in a similar way. This two-part process is iterated till mutual information converges to a local minimum.

\section{EXPERIMENTS AND RESULTS}

To quantitatively evaluate our novel approach we test it using several of the popular datasets in the community selected on basis of: popularity for benchmark testing; number of categories within the dataset; nature of the constituent visual categories. The visual feature descriptor utilized is SIFT (Lowe, 2004). We use k-means clustering for the purpose of partitioning feature space. A k-nearest neighbor $(\mathrm{kNN})$ classifier is used to evaluate the performance gain achieved by our approach over the standard BoW approach. A popular alternative classifier is the SVM. Our choice if motivated by the work in (Boiman et al., 2008), which defends k-NN vs. classifier like SVM; and use of k-NN in (Horster et al., 2008). In order to understand the performance of our approach with codebook size, we experiment with different topic codebook sizes. 


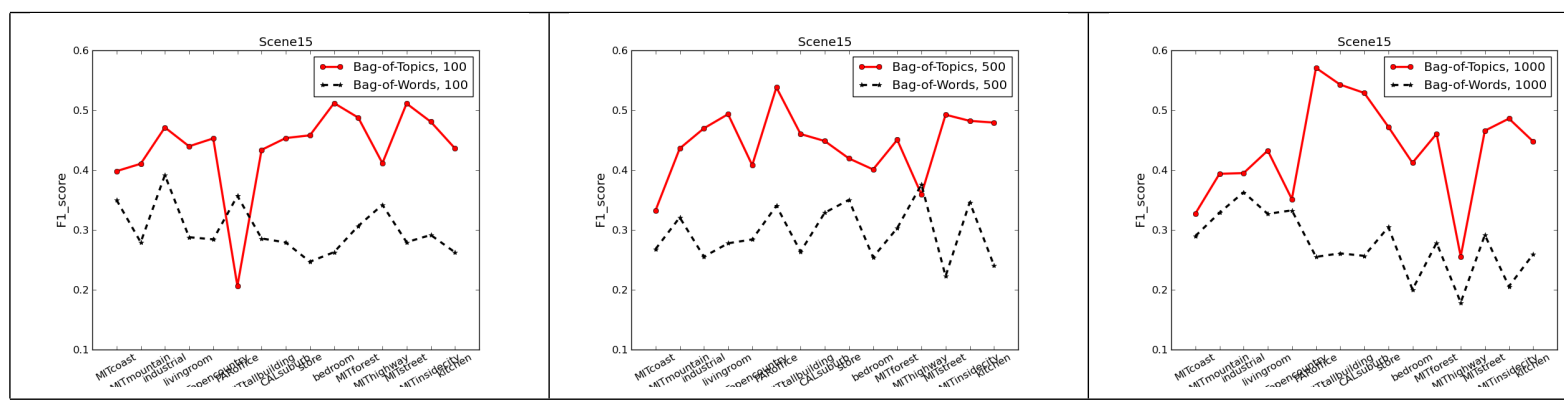

Table 1: Expt. Scene-15: Comparison of information theoretic co-clustering in terms of performance gain over the BoW model. The dataset utilized is Scene-15. The graphs show the $F_{1}$-score for each technique across the visual categories in the dataset. The codebook sizes are 100, 500, and 1000.

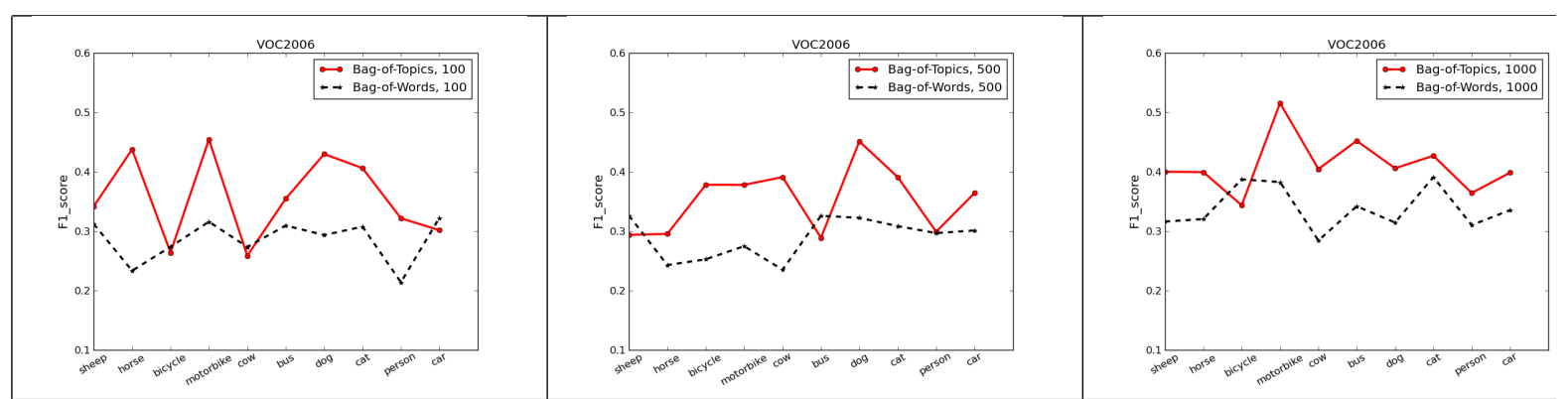

Table 2: Expt. Pascal VOC-2006: Comparison of information theoretic co-clustering in terms of performance gain over the BoW model. The dataset utilized is Pacal VOC-2006. The graphs show the $F_{1}$-score for each technique across the visual categories in the dataset. The codebook sizes are 100, 500, and 1000.

\subsection{Setup}

In this paper we are learning category specific codebooks. Negative training samples are collated from other categories in the dataset. The feature detector and descriptor SIFT detects on average a thousand interest points per image. PCA (Ke and Sukthankar, 2004) is used to project the feature space to 13 dimensions, based on a study of intrinsic dimensionality of visual descriptor data in (Gupta and Bowden, 2011). K-means clustering is utilized with Euclidean distance metric; randomly generated initial cluster centroids; and upper limit of 100 iterations. The k-NN classifier had a neighborhood size of 10. Classification performance was measured using $F_{1}$ score, which is the harmonic mean of precision and recall. It is commonly used for measuring document retrieval and classification performance.

$$
F_{1}=2 \cdot \frac{\text { precision } \cdot \text { recall }}{\text { precision }+ \text { recall }}
$$

We over-partition space to compute a huge codebook of 10000 words. We compute a word codebook of the same size as the topic codebook to be learned. We use 10 fold cross-validation in estimating the classification performance for both approaches. In all the graphs showing results of the experiments, the solid line is the $F_{1}$ score for our approach and the dashed line represents the BoW approach.

\subsection{Expt. Scene-15}

In this experiment we evaluate our approach against BoW for Scene-15 dataset. It has 15 visual categories of natural indoor and outdoor scenes. Each category has about 200 to 400 images and the entire dataset has 4485 images. Details can be found in (Fei-fei, 2005). We present results for different sizes of the topic codebook: $\{100,500,1000\}$. The results are shown in table 1 . The graphs show the $F_{1}$ score for different categories in Scene-15. The difference in $F_{1}$ scores between our approach and BoW is consistently large and remarkably better than it is for VOC-2006 and VOC-2007. This is an interesting result and alludes to the possibility that the nature of scene descriptor data is comparatively more amenable to our approach.

\subsection{Expt: Pascal VOC-2006}

In this experiment we evaluate our approach against BoW for Pascal VOC-2006 dataset. It is an early edition of the series of datasets used in the annual object categorization challenge. Several papers in literature 


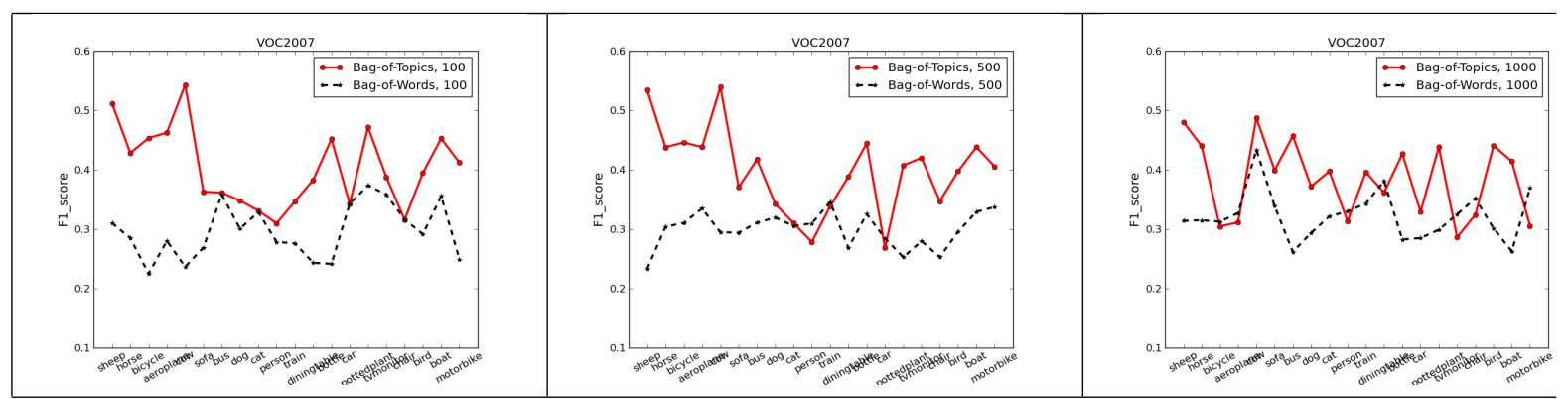

Table 3: Expt. Pascal VOC-2007: Comparison of information theoretic co-clustering in terms of performance gain over the BoW model. The dataset utilized is Pacal VOC-2007. The graphs show the $F_{1}$-score for each technique across the visual categories in the dataset. The codebook sizes are 100, 500, and 1000.

report results using this dataset. It has 10 visual categories with about 175 to 650 images per category. There are a total of 5304 images. Images do contain instances of multiple categories. Details can be found in (Everingham, ). We present results for different sizes of the topic codebook: $\{100,500,1000\}$. The graphs show the $F_{1}$ score for different categories in VOC-2006. The results are shown in table 2. Our approach performs significantly better than BoW for most of the categories and only slightly worse for a couple of categories.

\subsection{Expt: Pascal VOC 2007}

In this experiment we evaluate our approach against BoW for Pascal VOC-2007 dataset. It is the next edition in the Pascal series and has 20 categories and more images. The categories are in four themes: person; animal; indoor; vehicle. Each category contains images ranging from 100 to 2000, with 9963 images in all. Details can be found in (Everingham, 2007). We present results for different sizes of the topic codebook: $\{100,500,1000\}$. The graphs show the $F_{1}$ score for different categories in VOC-2007. The results are shown in table 3 . Our approach has performed remarkably better than BoW for all codebook sizes. The inherent difference between visual categories is reflected in the graphs which show considerable variation in $F_{1}$ score between different categories.

\subsection{Expt: Topic Codebook Size}

We want to understand the effect of the size of the topic codebook. Accordingly in this experiment we build topic codebook of several different sizes ranging from 50 topics to 5000 topics. These are extreme values and performance on them should provide a bounding limit on a viable topic codebook size. We evaluate our approach against BoW and we utilize the Pascal
VOC-2010 dataset. It is a compendium of previous editions in 2008 and 2009 as well a new images in 2010. It is considered a very challenging dataset. It has 20 visual categories with 300 to 3500 images per category. There are a total of 21738 images. Details can be found in (Everingham, 2010). The codebook sizes considered are: $\{50,100,500,1000,5000\}$. The results are collated in table 4 . The graphs show the $F_{1}$ score for different categories in VOC-2010.

\section{DISCUSSION}

We have presented an approach for discovering groups of descriptor vectors scattered in feature vector space. We argued that significant intra-category appearance variation causes descriptors sourced from the same object part to be scattered into different parts of feature space. The traditional approach of BoW in unable to combine these semantically equivalent but visually different descriptors into the same cluster. Consequently, the codebook of words fails to address the issue of intra-category variation. We analyzed the image-word joint distribution and used coclustering to find clusters with optimal joint distributions. Our technique assigned descriptors to topic based on both distribution in feature space and across images. The codebook of such topics is robust to intra-category variation and consequently performed better than BoW. Based on different codebook sizes, we found that a size in the range of 500 to 1000 provides the best performance. The margin of improvement is bigger for more difficult datasets which is an impressive result in favor of our approach.

\section{Acknowledgement}

This work was supported by the EPSRC project EP/H023135/1. 


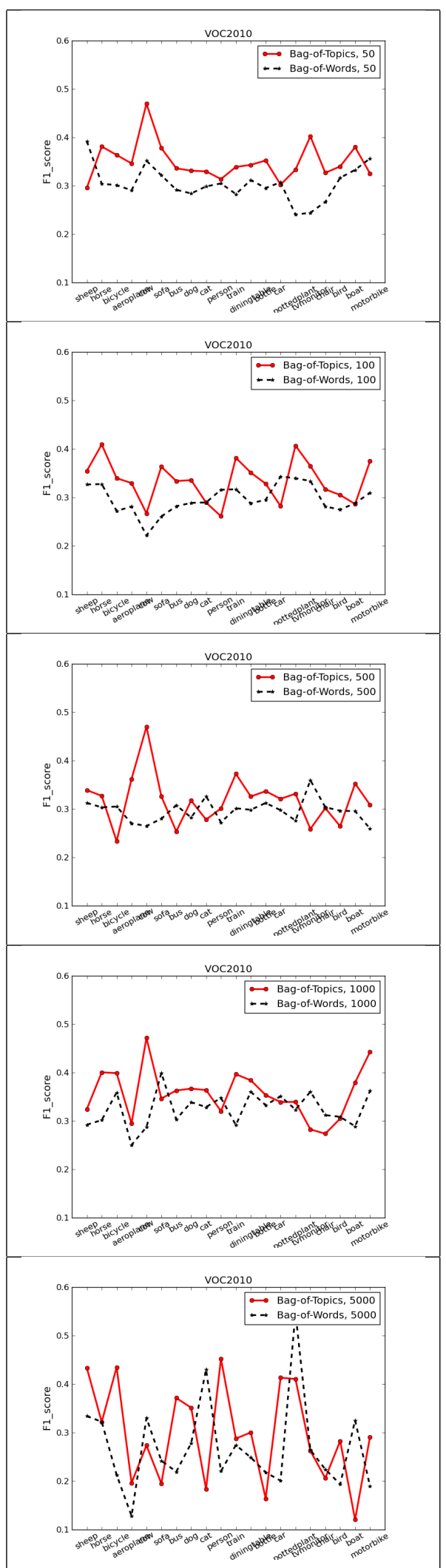

Table 4: Expt. Topic Codebook Size: Comparison of information theoretic co-clustering in terms of performance gain over the BoW model. The dataset utilized is Pacal VOC2010. The graphs show the $F_{1}$-score for each technique across the visual categories in the dataset. The codebook sizes are 50, 100, 500, 1000, and 5000 .

\section{REFERENCES}

Boiman, O., Shechtman, E., and Irani, M. (2008). In defense of nearest-neighbor based image classification. In CVPRO8, pages 1-8.

Cheng, Y. and Church, G. M. (2000). Biclustering of expression data. In Proc Int Conf Intell Syst Mol Biol. volume 8, pages 93-103.

Csurka, G., Dance, C. R., Fan, L., Willamowski, J., and Bray, C. (2004). Visual categorization with bags of keypoints. In In Workshop on Statistical Learning in Computer Vision, ECCV, pages 1-22.

Dhillon, I. S. (2001). Co-clustering documents and words using bipartite spectral graph partitioning. In Proceedings of the seventh ACM SIGKDD international conference on Knowledge discovery and data mining, KDD '01, pages 269-274, New York, NY, USA. ACM.

Dhillon, I. S., Mallela, S., and Modha, D. S. (2003). Information-theoretic co-clustering. In In $K D D$, pages 89-98. ACM Press.

Everingham, M. The PASCAL Visual Object Classes Challenge 2006 (VOC2006) Results. http://www.pascalnetwork.org/challenges/VOC/voc2006/results.pdf.

Everingham, M. (2007). The PASCAL Visual Object Classes Challenge 2007 (VOC2007) Results. http://www.pascalnetwork.org/challenges/VOC/voc2007/workshop/index.html.

Everingham, M. (2010). The PASCAL Visual Object Classes Challenge 2010 (VOC2010) Results. http://www.pascalnetwork.org/challenges/VOC/voc2010/workshop/index.html.

Fei-fei, L. (2005). A bayesian hierarchical model for learning natural scene categories. In In Proc. CVPR, pages 524-531.

Gupta, A. and Bowden, R. (2011). Evaluating dimensionality reduction techniques for visual category recognition using renyi entropy. In In Proc. of European Signal Processing Conf., pages 913-917.

Hartigan, J. A. (1972). Direct clustering of a data matrix. Journal of the American Statistical Association, 67(337):pp. 123-129.

Horster, E., Greif, T., Lienhart, R., and Slaney, M. (2008). Comparing local feature descriptors in plsa-based image models. In Proc. of the 30th DAGM symposium, pages 446-455, Berlin, Heidelberg.

Ke, Y. and Sukthankar, R. (2004). Pca-sift: a more distinctive representation for local image descriptors. In CVPR04, pages II: 506-513.

Liu, J. and Shah, M. (2007). Scene modeling using coclustering. In In Proc. ICCV, pages 1-7.

Lowe, D. (2004). Distinctive image features from scaleinvariant keypoints. International Journal on Computer Vision, 60(2):91-110.

Rycroft, C. H., Grest, G. S., Landry, J. W., and Bazant, M. Z. (2006). Analysis of granular flow in a pebblebed nuclear reactor. Physical Review E, 74. 